\section{Diffusion of Polymer-Coated Nanoparticles Studied by Fluorescence Correlation Spectroscopy}

\section{J iang J ohn Zhao, Sung Chul Bae, Feng Xie, and Steve Granick*}

Department of Materials Science and Engineering, University of Illinois, Urbana, Illinois 61801

Received J anuary 3, 2001

Revised Manuscript Received March 15, 2001

Introduction. Fluorescence correlation spectroscopy (FCS), an experimental method to extract information on dynamical processes from the fluctuation of fluorescence intensity, has enjoyed widespread application in chemical biology. ${ }^{1-3} \mathrm{~F}$ or example, it has been used to study binding of drugs to DNA molecules, ${ }^{4}$ to determine the density of macromolecules in biological systems, ${ }^{5}$ to measure the rate constants of chemical reactions, ${ }^{6}$ and so forth. The fluctuation of fluorescence is due to dynamic processes such as diffusion, aggregation, and chemical reactions, which occur in very small volumes $\left(\sim 10^{-15} \mathrm{~L}\right)$ in very dilute systems (from $\mathrm{pM}$ to $\left.\mathrm{nM}\right)$. By calculating the autocorrelation function $(G(\tau))$ of this fluctuation $(\mathrm{F}), \mathrm{G}(\tau)=\langle\delta \mathrm{F}(\tau) \delta \mathrm{F}(\mathrm{t}+\tau)\rangle\langle\delta \mathrm{F}(\mathrm{t})\rangle^{2}$, and by choosing a suitable model to analyze the autocorrelation function, the rate of the dynamic process is obtained. Compared to other techniques for studying diffusion problems, such as quasi-elastic light scattering, ${ }^{7}$ fluorescence recovery after photobleaching, ${ }^{8}$ laser-induced transient grating spectroscopy, ${ }^{9}$ and so forth, FCS presents unique advantages in measuring very dilute systems with high spatial resolution (down to the optical diffraction limit).

Apart from its popularity in biological and chemical sciences, FCS has only found application recently in the fields of materials science. A few works have been reported. For example, Sukhishvili et al. studied the center-of-mass diffusion of polymer molecules at the solid-liquid interface, ${ }^{10}$ and Akcakir et al. worked on number density and brightness of silicon nanoparticles. ${ }^{11}$ Here we report our FCS study on another type of material: colloidal suspensions. Understanding the dynamics of colloid particles, especially their interactions with their environments, is critically relevant to a large number of applications, such as ceramics, drug delivery, biosensing, food processing, and so forth. ${ }^{12}$ It is also an interesting and important topic at the level of fundamental scientific research. ${ }^{13}$ Here we study the interaction of charged colloidal particles and polyelectrolytes. ${ }^{14}$ The rate of Brownian motion of polystyrene nanoparticles is controlled by tuning their hydrodynamic radius through adsorption of a polyelectrolyte of opposite charge. FCS is successfully used to measure the diffusion coefficient of the particles. This study has initiated our further studies on the interaction of nanoparticles with different types of polymers and different surfaces.

Experimental Section. The FCS setup consists of three parts: light source, microscope, and data acquisition. Taking advantage of two-photon excitation of fluorescence, a femtosecond $\mathrm{Ti}$ :sapphire laser was adopted (Mai Tai, Spectra-Physics), which generated laser pulses

* Corresponding author. E-mail sgranick@iuc.edu. with fwhm (full width at half-maximum) of $100 \mathrm{fs}$ at a repetition rate of $80 \mathrm{MHz}$. The wavelength of the laser output was $800 \mathrm{~nm}$. A Zeiss inverted microscope (Axiovert S100TV, Carl Zeiss) served as the operational platform for the whole experiment, and an oil-immersed objective lens with a numerical aperture (NA) of 1.4 (Plan-APOCHROMAT, Carl Zeiss) was used. By introducing the laser beam through the objective lens, a very small excitation volume was generated within the sample solution. The lateral dimension of this excitation spot was about $0.3-0.4 \mu \mathrm{m}$, as determined by a calibration experiment using a well-studied dye, fluorescein (Aldrich), whose diffusion coefficient in water is known to be $\approx 300 \mu \mathrm{m}^{2} \mathrm{~s}^{-1}$. In this confocal geometry, the fluorescence from the sample was collected by the objective lens and was detected by a thermoelectrically cooled photomultiplier tube (PMT, Electron Tubes) at the bottom output port of the microscope. The output of the PMT was converted into photon counts by a preamplifier and a discriminator. The photon counting output was recorded by an integrated FCS data acquisiti on board and data acquisition, and data analysis was conducted by its software (ISS).

Polystyrene nanoparticles doped with fluorescent dye (Interfacial Dynamics Corp.) were chosen as the colloidal sample. They were negatively charged owing to surface sulfate groups, and the surface charge density (characterized by the supplier) was $1.5 \mu \mathrm{C} \mathrm{cm}^{-2}$. The counterion of the sulfate group is the hydrogen ion. The size of the particles was measured to be around $40 \mathrm{~nm}$ by scanning electron microscope (SEM) and also by static light scattering, with a standard deviation of the diameter (measured by the supplier) of 0.15 . In the experiments, the original polystyrene particle suspension was diluted $10^{4}$ times to form a final concentration of $3.0 \times 10^{11}$ particles $\mathrm{mL}^{-1}$. In this case, the particles can be regarded as isolated because their average spacing is about $3.0 \mu \mathrm{m}$, much larger than the Debye length $(0.6 \mu \mathrm{m})$.

Quaternized poly(vinylpyridine) (QPVP) was chosen as the polyelectrolyte adsorbate. The preparation of QPVP was by quaternization of poly(1,4-vinyl)pyridines (PVP) with ethyl bromide in the manner described previously. ${ }^{15}$ Narrow-distribution PVP samples with different molecular weights were quaternized to completion ( $98 \%$ as determined by infrared spectroscopy ${ }^{15}$ ), and the final weight-average molecular weight of QPVP $M_{w}$ $=35$ 100, 66 800, 158 100, and $253800 \mathrm{~g} \mathrm{~mol}^{-1}$ (Table 1). The concentration of all QPVP solutions was $0.2 \mathrm{mg}$ $\mathrm{mL}^{-1}$, and the final concentration of QPVP after addition to the particle suspension was $1.3 \mu \mathrm{g} \mathrm{mL}^{-1}$. The purpose of choosing this very low concentration was to eliminate the possible influence of the viscosity of the polymer solution on diffusion of the particles.

In experiments, a polystyrene particle suspension of $300.0 \mu \mathrm{L}$ was added into the sample cell and measured. Then 2.0 $\mu \mathrm{L}$ of QPVP solution was added, the mixture was stirred well, and new measurements were conducted. The precleaned chamber ed cover glass (L ab-Tek, Nunc) was chosen as sample cell. The $\mathrm{H}_{2} \mathrm{O}$ was purified by passage through Milli-Q (Millipore) deionizing and filtration columns. The ionic strength of the final suspension after addition of QPVP was $3 \times 10^{-6} \mathrm{M}$. The temperature was room temperature, $24-25^{\circ} \mathrm{C}$. 
Table 1. Characteristics of the Quaternized Poly(vinylpyridine) (QPVP)

\begin{tabular}{ccc}
\hline $\mathrm{M}_{\mathrm{w}}\left(\mathrm{g} \mathrm{mol}^{-1}\right)$ & $\begin{array}{c}\text { weight-average } \\
\text { charge ratio }\end{array}$ & $\begin{array}{c}\text { weight-average no. of } \\
\text { charges per chain }\end{array}$ \\
\hline 35100 & 0.4 & 170 \\
66800 & 0.7 & 325 \\
158000 & 1.7 & 770 \\
254000 & 2.7 & 1240
\end{tabular}

a Ratio of weight-average no. of charges per chain to the average charge per particle.

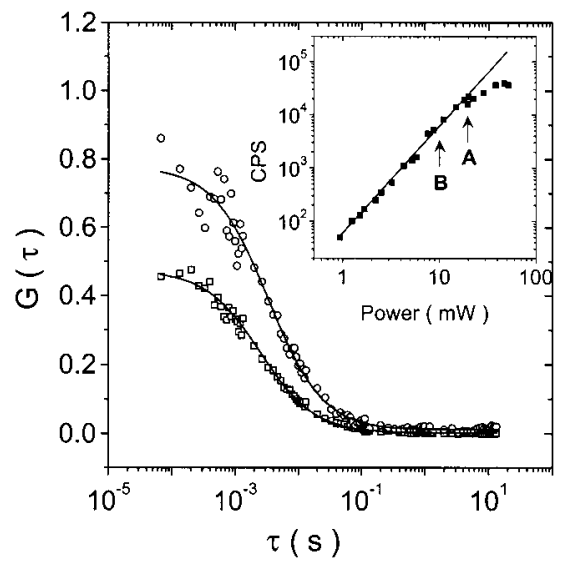

Figure 1. Autocorrelation function plotted as a function of time for pure polystyrene latex suspension at a concentration of $3.0 \times 10^{11}$ particles $\mathrm{mL}^{-1}(\square)$ and after addition of solution of QPVP with $M_{w}=66800 \mathrm{~g} \mathrm{~mol}^{-1}(\mathrm{O})$. Inset: the photon counts per second (cps) plotted vs the laser power at the sample stage on log-log scales. The quadratic dependence is shown by the solid line with a slope of 2 . Experiments were usually conducted at the power $\approx 18.0 \mathrm{~mW}$ (denoted by arrow A) to increase the photon counts. Measurements at lesser power ( $10 \mathrm{~mW}, \mathrm{~B})$ have been performed using an avalanche photodiode rather than a PMT, which gave identical results.

Results and Discussion. Figure 1 compares the time-dependent fluorescence autocorrelation function obtained with the pure particle suspension with that after adding QPVP solution of $\mathrm{M}_{\mathrm{w}}=66800$. The solid line shows the fit by a 3-dimensional Gaussian model for a self-diffusion process. The inset of Figure 1 demonstrates clearly the two-photon excitation in this experiment. It shows that when the laser intensity was below a certain level, the photon counts showed an excellent quadratic dependence on the laser power. Saturation occurred when the excitation light was too intense, and photobleaching was obvious when the laser power was even higher.

Sl ower particle diffusion after adding QPVP is clearly observed, as illustrated by diffusion coefficients of 9.8 and $6.0 \mu \mathrm{m}^{2} \mathrm{~s}^{-1}$ for pure particle and the QPVP-coated particles, respectively. The dependence of self-diffusion coefficient on molecular weight of QPVP is plotted in Figure 2. A continuous suppression of the rate of diffusion is observed.

Because of electrostatic attraction, the positively charged QPVP molecules will adsorb onto the polystyrene particles bearing groups of negative charge. As a result, the original polystyrene particle changes into a ball with a hard core coated with a wooly layer of QPVP. The behavior of the self-diffusion of an isolated particle can be characterized by the Stokes-Einstein equation, $\mathrm{D}=\mathrm{kT} / 6 \pi \eta \mathrm{R}$, where $\eta$ and $\mathrm{R}$ represent viscosity and radius of the particle, respectively. By taking the value of the viscosity of the water as $1.0 \mathrm{cP}$, the radius of the particles studied was obtained, and by subtracting the size of the polystyrene core, the thickness of the QPVP

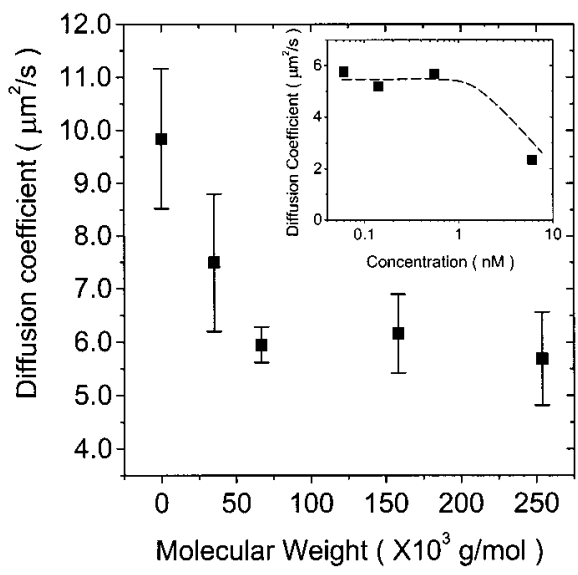

Figure 2. Diffusion coefficient plotted vs the molecular weight of the QPVP. The value of zero of $M_{w}$ represents the latex particles without added QPVP. Error bars represent the difference between the mean and the maximum deviation from the mean in at least 10-20 independent measurements. Inset: diffusion coefficient of polystyrene particles bearing adsorbed QPVP $\left(M_{w}=254000 \mathrm{~g} \mathrm{~mol}^{-1}\right)$ plotted against concentration of the particle suspension.

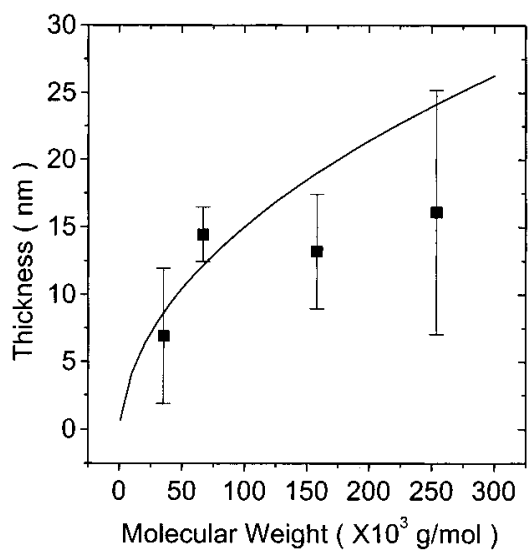

Figure 3. Thickness of the adsorbed QPVP layers plotted against molecular weight. Data are calculated from the selfdiffusion coefficient using the Stokes-Einstein equation. Line is estimated from eq 1 as described in text. Error bars represent the difference between the mean and the maximum deviation from the mean in at least 10-20 independent measurements.

on particles is obtained and plotted in Figure 3. (It should be noted that the "radius" here is a phenomenological parameter because the particle bearing adsorbed QPVP is no longer a "hard" ball.) The resulting augmentation of the hydrodynamic radius depends on the real thickness of the QPVP layer, which is also related to the conformation of the QPVP molecules. This is known to depend on the ionic strength of the solution and also on concentration of the polyelectrolyte. ${ }^{16}$

The inset of Figure 2 shows the results of measure ment of $D$ in particle suspensions of different concentrations varying by 2 orders of magnitude. Although a significant decrease of diffusion coefficient was observed at the highest concentration, possibly owing to aggregation of the particles, no dependence was observed in the range $0.06-0.55 \mathrm{nM}$, which is the range in which all the subsequent measurements in this study were performed. This allows us to rule out the conceivable mechanism of aggregation as an alternative explanation of the results presented below. Moreover, the data in the inset of Figure 2 illustrate the sensitivity of FCS for studying ultradilute systems. 
In the present study, the mean number of negative charges on each particle was around 470. For comparison, the mean number of positive charges per QPVP chain is listed in Table 1. As for relative concentrations, the ratio of the number of the particles and that of the QPVP molecules varied from 1:10 to 1:70, corresponding to QPVP with $M_{w}$ from 253800 to 35100 . Since the number of the QPVP molecules al ways exceeded that of the particles, it is reasonable to expect that the QPVP would not adopt a "flat" conformation when adsorbed even for the sample of lowest $M_{w}$, because of the competition in adsorption between the chains. This is easy to imagine for the samples of QPVP of chain lengths so high that the charge number of each chain exceeded that of the particle. It is also relevant for chains of lower $\mathrm{M}_{\mathrm{w}}$ since their molecular concentration was higher. Furthermore, hydrophobic interactions between the polymer chains also will serve to keep some parts of the polymer chain away from the particle surface. Another factor is evident when we consider the distribution of charges on the particle. The area each charge occupies is around $10.6 \mathrm{~nm}^{2}$, on average, giving an average charge spacing of $4 \mathrm{~nm}$, which is comparable to the radius of gyration $\left(R_{g}\right)$ of the QPVP of low molecular weight. The surface curvature of the particle is expected to enhance this effect. From all of these points of view, it seems more natural for the QPVP to take a "fluffy" conformation than the "flat" one that would be expected, at these dilute concentrations, when chains adsorb to a planar surface.

For these reasons we tentatively hypothesized that the QPVP molecules of different molecular weight adopted similar adsorbed conformations. As an estimate of the adsorbed layer thickness from which to begin, we refer to $R_{g}$ of QPVP. The $R_{g}$ of a stiff chain can be written as

$$
\begin{aligned}
\left\langle R_{g}{ }^{2}(L)\right\rangle=\frac{1}{3} L_{P} L- & L_{P}^{2}+ \\
& \frac{2 L_{P}^{3}}{L}\left[1-\frac{L_{P}}{L}\left(1-\exp \left(-L / L_{P}\right)\right)\right]
\end{aligned}
$$

where $L$ and $L_{p}$ are the contour length and persistence length of the polyelectrolyte chain, respectively. ${ }^{17}$ Since there appears to be no report of the values of these parameters for QPVP, the needed data were chosen for a similar flexible polyelectrolyte, poly(styrenesulfonate), adopting the value of monomer length $(\alpha)$ and $L_{p}$ as 0.255 and $1.4 \mathrm{~nm}$, respectively. 18,19 This gives $2 \mathrm{R}_{\mathrm{g}} \approx 8.6$, 12,19 , and $24 \mathrm{~nm}$ for the molecular weights (in ascending order) used in the present study. Figure 3 shows that the layer thickness determined by FCS based on the Stokes-Einstein equation compares favorably to these estimates.

A few words are necessary about uncertainties in the experiment. After addition of the QPVP, extremely high photon counts were recorded from time to time in the measurement, which can be attributed to clusters of particles entering the excitation volume due to the aggregation induced by the adsorbed QPVP. Also, polydispersity of the particles contributed to the scatter in the diffusion coefficient data, as clearly seen from that for the bare particle $\left(\mathrm{M}_{\mathrm{w}}=0\right.$ in Figure 2). The QPVP al so caused the particles to adsorb onto the walls of the sample cell made of polystyrene due to hydrophobic interactions, as well as sedimentation down to the bottom of the sample cell. This was evidenced by the
100 times higher photon counts recorded when the focal point of the laser was adjusted onto the surface of the sample cell. This also resulted in a decrease of the population of the particles in solution and, as a result, an increase of the $\mathrm{G}(0)$ value in Figure 1 . Therefore, only those data without such big shots of photon counts were accepted. However, particle aggregation did contribute to data scatter. Another influence could be an optical trapping effect by the strong laser beam used in this study because of the limited sensitivity of our PMT.20 A more detailed study by using a more sensitive detector, an avalanche photodiode, is in progress, which will allow experiments using lower laser power and more dilute suspensions.

It is interesting to reflect on the fact that the size of the latex particles was comparable to that of the QPVP molecules in solution. The real thickness of the adsorbed QPVP layers should in principle differ from the dimensions of the "free" molecules and al so from the thickness of QPVP adsorbed onto a planar surface. This in turn raises intriguing questions: What does the QPVP molecule look like when it is next to a hard ball of comparable dimension but opposite charge? How does this contribute to the hydrodynamic radius of the hairy ball? F urthermore, it will be very interesting to see the behavior of this system in different ionic environments as well as at different temperatures. Apparently, the extremely sensitive FCS technique has opened up a new road to access these questions, and further studies are in progress.

Acknowledgment. The authors thank $Y$. Chen and J . Müller (University of Minnesota) and P. So (MIT) for their invaluable advice in constructing the FCS setup and the group of Charles F. Zukoski for light scattering measurement. This work was supported by the U.S. Department of Energy, Division of Materials Science, under Award No. DEFG02-ER9645439, through the Frederick Seitz Materials Research Laboratory at the University of Illinois at Urbana-Champaign.

\section{References and Notes}

(1) Maiti, S.; Haupts, U.; Webb, W. W. Proc. Natl. Acad. Sci. U.S.A. 1997, 94, 11753.

(2) Widengren, J .; Rigler, R. Cell. Mol. Biol. 1998, 44, 857.

(3) Berland, K. M.; So, P. T. C.; Chen, Y.; Mantulin, W. W.; Gratton, E. Biophys. J . 1996, 71, 410 and references therein.

(4) Magde, D.; Elson, E.; Webb, W. W. Phys. Rev. Lett. 1972, 29, 705 .

(5) Weissman, M.; Schindler, H.; Feher, G. Proc. Natl. Acad. Sci. U.S.A. 1976, 73, 2776.

(6) I cenogle, R. D.; Elson, E. L. Biopolymers 1983, 22, 1919.

(7) Pecora, P., Ed. Dynamic Light Scattering: Applications of Photon Correlation Spectroscopy; Plenum Press: New Y ork, 1985.

(8) Tseng, K. C.; Turro, N. J .; Durning, C. J . Phys. Rev. E 2000, 61, 1800.

(9) Eichler, H. J .; Gunter, P.; Pohl, D. W. Laser-Induced Dynamic Gratings; Springer-Verlag: Berlin, 1986.

(10) Sukhishvili, S. A.; Chen, Y.; Müller, J . D.; Gratton, E.; Schweizer, K. S.; Granick, S. Nature 2000, 406, 146.

(11) Akcakir, O.; Therrien, J .; Belomoin, N.; Barry, N.; Muller, J. D.; Gratton, E.; Nayfeh, M. Appl. Phys. Lett. 2000, 76, 1857.

(12) Russel, W. B.; Saville, D. A.; Schowalter, E. R. Colloidal Suspensions; Cambridge University Press: Cambridge, 1989.

(13) Luo, L. S.; Phillies, G. D. J . Phys. Rev. E 1995, 51, 43.

(14) Caruso, F.; Lichtenfeld, H.; Giersig, M.; Möhwald, H. J . Am. Chem. Soc. 1998, 120, 8523.

(15) Sukhishvili, S. A.; Granick, S. J . Chem. Phys. 1998, 109, 6861. 
(16) Fleer, G. J .; Cohen Stuart, M. A.; Scheutjens, J . M. H. M.; Cosgrove, T.; Vincent, B. Polymers at Interfaces; Chapman \& Hall: London, 1993.

(17) Dautzenberg, H.; J aeger, W.; Kotz, J .; Philipp, B.; Seidel, Ch.; Stscherbina Polyel ectrolytes: Formation, Characterization, and Application; Hanser: Munich, 1994.
(18) Eisenberg, H. Acta Polym. 1998, 49, 534.

(19) Borochov, N.; Eisenberg, H. Macromolecules 1994, 27, 1440.

(20) Grier, D. G. Curr. Opinion Colloid Interface Sci. 1997, 2, 264

MA0100145 